\title{
Spectral dependence of the photomagnetic effect in porous silicon
}

\author{
O.V. Vakulenko, S.V. Kondratenko \\ Taras Shevchenko Kyiv Univ., 6 Glushkova Prosp., 03127 Kyiv, Ukraine \\ e-mail:kondr@mail.phys.univ.kiev.ua.
}

\section{B.K. Serdega}

Institute of Semiconductor Physics NASU, pr. Nauki, 45, Kyiv, 03028

e-mail: bshl@polarget.semicond.kiev.ua

\begin{abstract}
Measurements of the photomagnetic effect spectra of PS/c-Si were carried out. Obtained results were explained by influence of the spatial charge region of the heterojunction between wide-band porous silicon and $c$-Si substrate of the $n$-type. The form of the PME spectrum in the long-wave region indicates to the drift of the nonequilibrium charge carriers in the $c$-Si to the PS/c-Si boundary, that is presence of the increased spatial charge layer in $c$-Si. Relatively high value of the PME in the strong absorption region of the porous silicon indicates to the presence of the PME component caused by porous silicon material.
\end{abstract}

Keywords: photomagnetic effect, porous silicon, heterojunction.

Paper received 26.03.01; revised manuscript received 08.04.01; accepted for publication 13.07.01.

\section{Introduction}

The structures with porous silicon layers have a great potential for their application in optoelectronics. However, the investigation of such photoelectric properties as photoconductivity and photomagnetic effect has not received sufficient attention as compared with the optical and luminescent ones. Particularly, the research of mentioned photoelectric properties is important for studying the peculiarities of the nonequilibrium charge carrier transport, which is necessary for design of the photosensitive and electroluminescent devices with the porous silicon layer.

It is common knowledge that the heterojunction between crystalline substrate and porous silicon (PS) is of considerable importance in photoelectric properties of the structures with porous silicon layer $[1,2]$. The commonly adopted energy diagram of the heterojunction takes into account the pointed up band bending in the PS layer and $p$-type crystalline silicon substrate [3,4], which corresponds to the presence of the depleted region near the boundary both in the porous silicon and in the substrate. On the assumption that the similar energy structure might be inherent to the sample surface which is the substrate, the investigation of the photomagnetic effect is quite actual. Many researches were devoted to comprehensive study of this effect in homogeneous materials and in different non-homogeneous structures. Moreover, these structures are considered to be materials with gradient either of the potential or band gap width in the direction of the light spreading. As it was found in [5-7], the spectral dependencies sensitive to the value and sign of the band bending have features associated with such kinds of structures. When considering its interpretation to be unique and experienced, the testing of the energy structure on the boundary between PS and substrate became possible by means of measuring the photomagnetic voltage. What is more, taking into account the only known work devoted to this problem [8], it should be expected revealing the photomagnetic voltage caused by PS.

Therefore, the aim of our paper is to investigate the photomagnetic effect in the structures with porous silicon layers in the intrinsic absorption region of both materials. Moreover, the stationary conditions have been provided as distinguished from [8], where the impulse method and the power laser emission have been applied. And although the condition of linear excitation is not completely provided, at least, its probability is increased, 


\section{O.V. Vakulenko et al.: Spectral dependence of the photomagnetic effect in porous ...}

which simplifies the interpretation of results. But the weak intensity of the excited illumination, as compare to the laser method, and the low values of the charge carriers mobility $\left(10^{-2}-10^{-3} \mathrm{~cm}^{2} / \mathrm{V} \cdot \mathrm{s}\right)$ which are indicated somewhere [9], make it difficult to observe the photomagnetic effect in such conditions. The argument in support of this statement is absence of any publications concerning this question. At the same time, the high information capacity of the photomagnetic voltage method about the kinetic parameters of the material porous silicon, (as in our case) and the features of the charge carriers transport, the information about which is limited in spite of large amount of references.

\section{Experiment}

The PS layer were formed by anodization of (100) n-type crystalline silicon with a resistivity of $500 \Omega \cdot \mathrm{cm}$ and thickness $d=4 \cdot 10^{-2} \mathrm{~cm}$ in $\mathrm{C}_{2} \mathrm{H}_{5} \mathrm{OH}: \mathrm{HF}$ dissolution (1:1) with current density $25 \mathrm{~mA} / \mathrm{cm}^{2}$ during $3 \mathrm{~min}$. The porous silicon thickness was about $18 \mu \mathrm{m}$. After anodization, the point contacts with the diameter $1 \mathrm{~mm}$ were formed on the porous silicon surface.

Measurements of spectral dependencies of the photomagnetic effect (PME) and photovoltage were carried out at the temperature $300 \mathrm{~K}$ under illumination of the porous silicon layer. The contacts were not illuminated in these experiments. The monochromator MDR12 with $70 \mathrm{~W}$ halogen lamp served as a source of light, which provides the value of the exciting illumination intensity about $10^{14}$ quanta/ $\mathrm{cm}^{2} \cdot \mathrm{s}$. The value of the magnetic field was about $3.2 \cdot 10^{5} \mathrm{~A} / \mathrm{m}$, and the $\mathrm{PME}$ value linearily depends on the magnetic field value. Moreover, the linear dependence of the PME and photovoltage signals on the exciting illumination intensity took place in the all spectral region, which enabled us to normalize the obtained spectra to the constant number of the exciting illumination quanta which crossed over the front surface of the structure. It is worth to note that the lateral Wallmark photovoltage [10] takes place under modulated illumination of the sample surface. It should be taken into account when measuring the photomagnetic voltage. Therefore, the value of the latter was determined as the difference between the photovoltage signals with switched on and switched of magnetic fields. Taking into consideration that the magnetic field under certain condition might influence on the photovoltage value, and the origin of this effect could not be associated with the diffusion of the nonequilibrium charge carriers, so it might distort the true PME value, the determination of the later was carried out from the measurement of two opposite directions of the magnetic field.

\section{Results and discussion}

In the Fig. 1 spectral dependencies of the photomagnetic voltage in the structure with the porous silicon layer (curve 1) and the crystalline silicon sample before the anodi- zation (curve 2) are shown. Let us to point out that the sign of both signals corresponds to the normal component of the PME. This means that the PME signal in both cases is formed by the nonequilibrium charge carrier flows in the PS layer and in the c-Si substrate in the direction of the light spreading (as for the curve 2, at least in the most part of it).

Let focus the attention on the detail analysis of the curve 1 . The presence of the considerable value of the photomagnetic voltage in the spectral region with the low gradient of the nonequilibrium charge carriers caused by weak absorption ( $\mathrm{ad} \sim 0.04$ at $h v=1.1 \mathrm{eB}[11])$, when the diffusion current is negligible, indicates to existence of the drift of the nonequilibrium charge carriers in the spatial charge region of the $c$-Si substrate in the direction to the PS/ c-Si boundary. It is well known that such drift observed when the band bending in the $n$-type material pointed down, that is in the case characteristic of the layer enriched by majority charge carriers. The spectral dependence of PME must be shifted to the short-wave region relatively to the optical absorption spectra on condition the lack of the band bending, because when the absorption coefficient is small, the diffusion component and, consequently, the PME signal are practically absent.

Such shift is observed when the photomagnetic effect is investigated in the $c$-Si substrate before the creation of the porous silicon layer (curve 2, Fig. 1). The PME spectrum of the crystalline silicon, namely the considerable fall in the short-wave region, indicates to the presence of the band bending caused by the spatial charge region depleted by the majority charge carriers. In such a case, the drift of the nonequilibrium charge carriers takes place in the direction to the illuminated surface. As a result, the negative component of the PME signal arises, and its value becomes more greater, when the larger light part is absorbed in the spatial charge layer, which leads to the PME spectrum fall in the short-wave region.

The form of the PME spectrum of the structure with the porous silicon layer can be explained by models which foresee the existence of the heterojunction between $c-\mathrm{Si}$

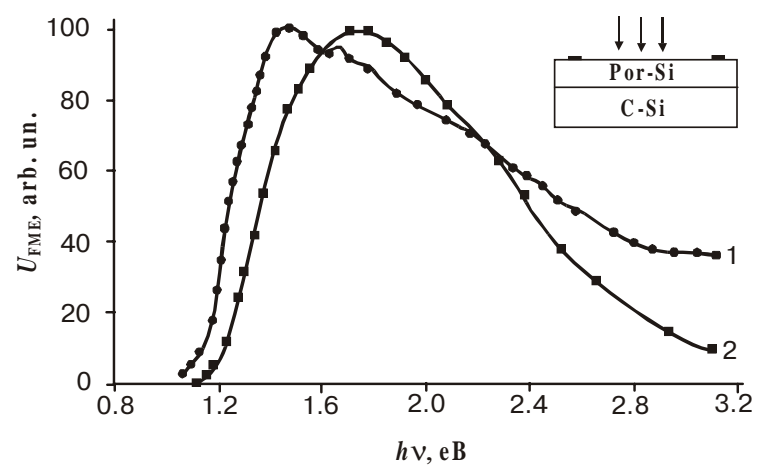

Fig. 1. The spectral dependencies of the PME for the structure with the porous silicon layer (curve 1) and crystalline silicon substrate (curve 2). a) A scheme of the sample investigated. 


\section{O.V. Vakulenko et al.: Spectral dependence of the photomagnetic effect in porous ...}

substrate and wide-gap PS layer. As follows from the analysis of the spectral dependencies the band gap width $E_{g}^{P S}$ is about $1.6 \mathrm{eB}$. In the spectral region $h v>E_{g}^{P S}$ the absorption in porous silicon should be taken into account. So, when the energy of quanta is $h v=1.2 \div 1.6 \mathrm{eB}$, the main contribution in to the PME signal is made by the $c$ Si substrate, and the nonequilibrium charge carriers absorbed in the porous silicon do not contribute to the PME signal. The absorption in porous silicon increases with increasing the quantum energy. As a result, the contribution of the $c$-Si substrate in the general PME signal decreases. It is well known that the PME spectrum falling in the region of the strong absorption is practically absent if the field component of the PME signal is caused by the drift of nonequilibrium charge carriers from the illuminated surface. For such case is realized when the radiation is absorbed in the single crystalline silicon substrate of the PS/ $c$-Si structure, then the contribution of $c$-Si in the general PME signal can be estimated using the spectral dependence of absorption of a «free» porous silicon layer as known from [12]. The result of similar calculations is shown in the Fig. 2 (curve 1).

Relatively large value of the photomagnetic voltage in the short-wave region (where porous silicon has the strong absorption) corresponds to the PME component formed in the porous silicon layer and having the same sign as in the $c$-Si substrate. The difference between the value of photomagnetic voltage of the PS/ $c$-Si structure (curve 2) and the calculated contribution of the $c$-Si substrate (curve 1) is caused by the PME component formed in PS layer (Fig. 3).

The normal sign of the photomagnetic voltage formed in PS layer is caused by the flux of the nonequilibrium charge carriers towards the boundary PS/ $c$-Si. In the general case, the flux of the nonequilibrium charge carriers consists of the diffusion and field components. The diffusion component appears under conditions of the existence of a nonequilibrium charge carrier gradient. It is

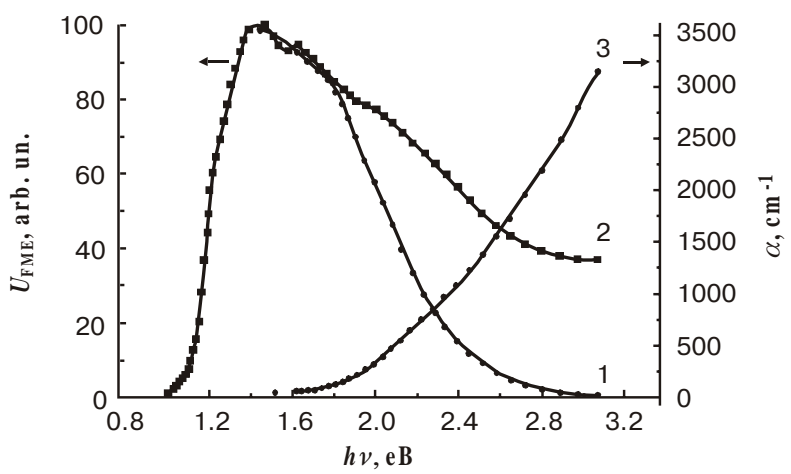

Fig. 2. The PME spectrum of the PS/c-Si structure (curve 2), calculated values of the contribution of the crystalline silicon substrate into the general PME signal (curve 1), and the spectral dependence of the absorption coefficient of the porous silicon layer (curve 3) [12].

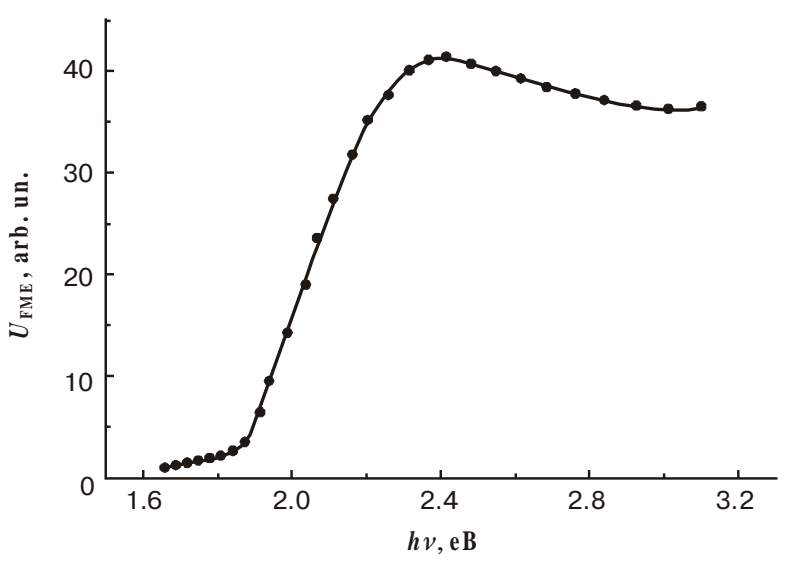

Fig. 3. The spectral dependence of the PME component that is formed in the PS layer.

known that such gradient takes place only in the region of a strong absorption. Relative contribution of the diffusion component grows with increasing the absorption coefficient, that is with decreasing of the wave length of the exciting illumination. When the absorption is small, and generation is consequently more uniform (in the region 1.4-1.6 eB), the general contribution in the nonequilibrium charge carriers current is given by the field component. The field component is caused by an ambipolar drift of the nonequilibrium charge carriers in the spatial charge region of the PS layer near the PS/ $c$-Si boundary to the $c$-Si substrate. It is well known that the pointed direction of the drift is possible only in the case of depletion of band bending. As a result, the following energy diagram of the heterojunction between the porous silicon layer and $c$-Si substrate can be proposed (Fig. 4a). Here, more wide - band porous silicon $\left(E_{g}^{P S} \sim\right.$ $1.6 \mathrm{eB})$ behaves as $p$-type semiconductor with pointed down band bending near the PS/ $c$-Si boundary. The as-

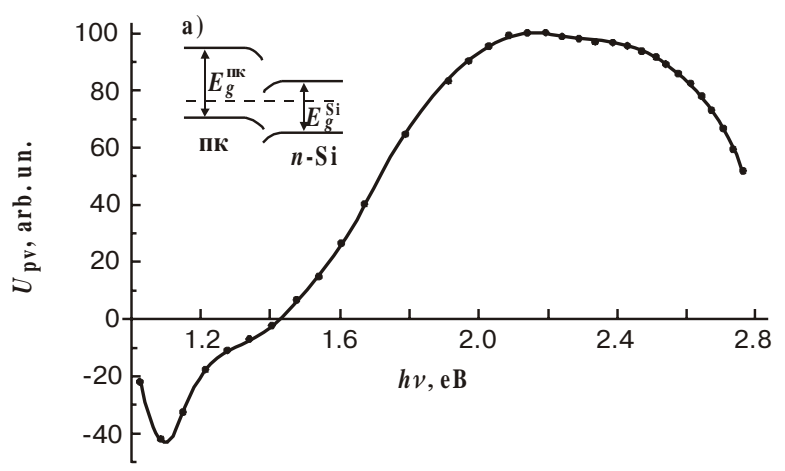

Fig. 4. The spectral dependence of the photovoltage for the structure with the porous silicon layer. a) A schematic band diagram. 


\section{O.V. Vakulenko et al.: Spectral dependence of the photomagnetic effect in porous ...}

sumption about the difference between the conductivity type of the PS and $c$-Si substrate is in a good agreement with the results obtained by other methods $[13,14]$.

The spectral dependence of the lateral photovoltage of the structure with the porous silicon layer (Fig. 4) testifies the proposed energy diagram. The obtained spectral dependence can be explained as follows. The photovoltage in the long-wave region caused by separation of the electron-hole pairs in the spatial charge field of the $c$ Si substrate. With increasing the quantum energy, the absorption in the PS layer takes place. The change of the photovoltage sign caused by opposite direction of the spatial charge field in the PS layer near the PS/ $c$-Si boundary relatively to $c$-Si substrate is therewith observed.

The relative by slow growth of the photovoltage spectrum in the region $h v=1.6 \div 2 \mathrm{eB}$, when the absorption in the porous silicon material increases while the number of quanta absorbed in the spatial charge region decreases, indicates to formation of the photovoltage component in the porous silicon bulk. Evidently, such photovoltage may be caused by not-uniformity of the sample or/ and the presence of the inner electric field. It should be pointed out that the field component of the PME formed in the porous silicon may be caused by the drift of the nonequilibrium charge carriers in the inner electric field, presence of which was found by author [16] when studying the electronic properties of the PS under illumination and adsorption of the ammonium.

\section{Conclusions}

The photomagnetic voltage has been found in the PS/c-Si structure by modulating of the excited illumination with a low intensity. This voltage generated in the crystalline substrate were observed in the transparency region of the porous silicon. The PME spectrum formed in this region evidences to presence of the increased band bending in the $c$-Si substrate near PS/ $c$-Si boundary. Relatively high value of the PME signal in the short-wave region indicates to formation of the PME component caused by PS material. It was found that the depleted spatial charge region presence in the PS material near PS/c-Si boundary, which causes the drift of the nonequilibrium charge carriers in the PS to wards the $c$-Si substrate.

The main result of the work is in separation of the PME component generated in the porous silicon bulk using the spectral dependence of the absorption for typical porous silicon layers obtained by standard method. Moreover, its form testifies both drift and diffusion origin of this effect. To obtain quantitative results that may be calcu- lated from this dependence, it is necessary to know the absorption parameters of specific samples or carry out measurements of the PME on the «free» PS layer.

\section{References}

1. E.V. Astrova, A.A. Lebedev, A.D. Remenyuk, V.Yu. Rud', Yu. V. Rud'. Photosensitivity of silicon - porous silicon heterostructures (in Russian) // Fiz. Tekhn. Poluprov. 31(2), pp. 159-161 (1997)

2. V.Yu. Rud', Yu. V. Rud'. Photoelectric properties of porous and single crystal silicon heterocontacts (in Russian) // Fiz. Tekhn. Poluprov. 31(2), pp. 245-248 (1997).

3. S.V. Svechnikov, E.B. Kaganovich, E.G. Manoilov. Photosensitive porous silicon based structures // Semiconductor Physics, Quantum Electronics \& Optoelectronics. 1(1), pp. 13-17 (1998).

4. L.A. Balagurov. Porous silicon. Obtaining, properties, possible applications (in Russian) // Materialovedenie. 3, pp. 2345 (1998).

5. A.V. Buyanov, S.G. Gasan-zade, I.P. Zhadko et al. O spectralnoj zavisimosti fotoprovodimosti i fotomagnitnogo effecta $v$ epitaxialnykh sloyakh $\mathrm{Cd}_{\mathrm{x}} \mathrm{He}_{1-\mathrm{x}} \mathrm{Te} / \mathrm{CdTe} \mathrm{s}$ anodno okislennoi svobodnoi poverhnostju (in Russian) // Fiz. Tekhn. Poluprov. 26(4), pp. 629-635 (1992).

6. R.S. Gabarev, V.A. Kakuhov, S.I. Chikachev. Osobennosti fotomagnitnogo effecta $v$ varizonnykh structurakh $\mathrm{GaAs}_{1-\mathrm{x}-\mathrm{y}} \mathrm{Sb}_{\mathrm{x}} \mathrm{P}_{\mathrm{y}}$ (in Russian) // Fiz. Tekhn. Poluprov. 19(4), pp. 742-744 (1985).

7. R.S. Gabarev, A.F. Kravchenko, B.V. Morozov. Spectralnaya zavisimost fototoka v varizonnykh poluprovodnikakh (in Russian) // Fiz. Tekhn. Poluprov. 17(9), pp. 1588-1591 (1983).

8. E. Shatkovskis, J. Vercinski, J. Jagminas. Nonradiative and radiative recombination of charge carriers in porous silicon under intense laser excitation // Phys. stat. sol. (a) 165, pp. 231-238 (1998).

9. N.S. Averkiev, L.P. Kazakova, E.A.Lebedev, N.N. Smirnova. Charge carrier drift mobility in porous silicon (in Russian) // Fiz. Tekhn. Poluprov. 35(5), pp. 609-611 (2001).

10. D.W. Boeringer, R. Tsu. Lateral photovoltaic effect in porous silicon // Appl. Phys. Lett. 65(18), pp. 2332-2334 (1994).

11. W.C.Dash, R.Newman. Intrinsic optical absorption in single crystal Ge and Si at 77 and $300 \mathrm{~K} / /$ Phys. Rev. 99(4), pp. 1151-1155 (1955).

12. L.V. Belaykov, D.N. Gorachev, O.M. Sreselly, I.D. Yaroshetsky. Svetochuvstvitelnyye structuri Shottki na poristom kremnii (in Russian) // Fiz. Tekhn. Poluprov. 27(8), pp. 1371-1374 (1993).

13. L. Burstein, Y. Shapira, J. Partee, J. Shinar, Y. Lubianiker, I. Balberg. Surface photovoltage spectroscopy of porous silicon // Phys.Rev.B. 55(4) pp. 1930-1933 (1997).

14. E.F. Venger, E.B. Kaganovich, S.I. Kirilova, E.G. Manoilov, V.E. Primachenko, S.V. Svechnikov. Porous silicon / silicon structures investigation by temperature dependence of photovoltage method (in Russian) // Fiz. Tekhn. Poluprov. 33(11), pp. 1330-1333 (1999).

15. Yu.I. Ravich, Fotomagnitnyi effekt $v$ poluprovodnikakh $i$ yego primenenie (in Russian) (Photomagnetic effect in semiconductors and its application). M.: Sov. radio, 1967. P. 96.

16. Yu.A. Vashpanov. Elektronnyye svoistva mikroporistogo kremniya pri osveshchenii i adsorbtsii ammiaka (in Russian) // Pisma v ZTF. 23(11), pp. 77-82 (1997). 